\title{
Impact of Modern Drug Therapy on Surgery: Ulcerative Colitis
}

\author{
Florian Kuehn ${ }^{a}$ Richard A. Hodin ${ }^{b}$ \\ ${ }^{a}$ Department of General, Visceral and Transplant Surgery, Ludwig-Maximilians-University Munich, Munich, Germany;

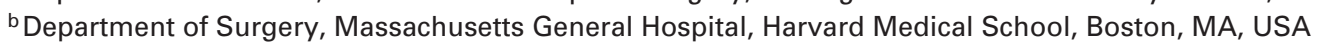

\section{Keywords}

Ulcerative colitis · Surgery · Biologicals ·

lleal pouch-anal anastomosis, IPAA

\section{Summary}

Background: The primary treatment of ulcerative colitis (UC) is conservative, and substantial therapeutic progress has been made in the past few decades. Meanwhile, biologicals have become a mainstay in the treatment for steroid-refractory UC. Despite further development of drug therapy and an increased time span to operation, a significant proportion of patients with UC require surgical intervention. Surgical intervention needs to be carried out in medically refractory cases, imminent or malignant transformation, or complications. This article discusses the impact of modern drug therapy on surgery for UC. Methods: A selective literature search of PubMed was conducted, taking into account current studies, reviews, meta-analyses, and guidelines. Selected articles were then reviewed in detail and recommendations were drafted based on data and conclusions of the articles. Results: In recent years, modern drug therapy has changed the timing, approach, and outcomes of surgery for UC. Most of the studies showed a decrease in surgery rates over time while the rate of emergency colectomies remains unchanged. So far, no convincing surgery-sparing effect of newer medications has been established, and it remains debatable if surgery rates have decreased because of improved management for UC in general or due to the introduction of

Data from this manuscript have in part been presented at the symposium 'Frontiers in Surgical Evolution' on September 21, 2018 in honor of Prof. Dr. Ernst Klar, Rostock, Germany. biologicals. The intensified conservative therapy with increasing use of biologics has been accompanied by a trend towards performing a three-step procedure in the last decade. There is a subset of patients with complex refractory disease who most likely benefit from elective surgery as an alternative to prolonged conservative therapies after failure of first-line treatment. The majority of patients after ileal pouch-anal anastomosis can avoid hospitalizations and colitis-related medications with their associated potential adverse effects. In addition, the procedure substantially reduces UC-related symptoms and the risk for dysplasia or cancer. There is a long-term pouch success rate of $>90 \%$ after 10 and 20 years of follow-up. Conclusion: Conservative medical therapy in the treatment of UC will continue to develop and the number of approved therapeutics will grow. Surgery should not be considered as the negative endpoint of treatment modalities but as a good alternative to a prolonged conservative therapy for some patients. In conclusion, a close cooperation between the various disciplines in the pre- and postoperative management is essential in order to optimize the timing and outcome of patients with UC.

(C) 2018 S. Karger GmbH, Freiburg

\section{Introduction}

Inflammatory bowel diseases (IBD) are multifactorial chronic diseases. The primary treatment of IBD is conservative (medical), and decisions regarding surgery should always be made in the context of a multidisciplinary care model. Despite further development of drug therapy and an increased time span to operation [1], a significant proportion of patients with ulcerative colitis (UC) require surgical intervention. Approximately $20-35 \%$ of patients

\section{KARGER}

() 2018 S. Karger GmbH, Freiburg

Fax +497614520714 
with UC will undergo surgery during the course of their disease [2, 3]. The fulminant form of UC is characterized by mortality rates between 27 and 57\% in case of primary treatment failure [3]. Restorative proctocolectomy with ileal pouch-anal anastomosis (IPAA) has become established as a standard surgical treatment for UC over the past 30 years. Ileo-anal pouch surgery provides the best possible quality of life for the patient with an average of 5-6 bowel movements per day and maintenance of continence in over $90 \%$ of patients [4-8]. The operation can be carried out as a one-, two- or three-stage procedure - depending on the individual risk profile of the patient. In recent years, modern drug therapy has changed the timing, approach, and outcomes of surgery for UC. In 2005, the chimeric monoclonal anti-TNFa antibody infliximab was approved as rescue therapy for patients with refractory UC. Since then, other anti-anti-TNF antibodies followed and new classes of biologicals with new target mechanisms such as vedolizumab (an integrin receptor antagonist) have already been approved for the treatment of patients with UC refractory to infliximab. In the future, combination therapies with different biological agents and other novel medications will further increase the modulation of our complex immune system. It has become critical that surgical management accounts for risks that accompany patients under immunosuppressive or immunomodulatory therapy. The current article summarizes how modern drug therapy has influenced the surgical therapy of UC.

\section{Medical Treatment for Ulcerative Colitis}

Medical treatment ranges from probiotics to calcineurin inhibitors and needs individual adjustment for each patient. Mild-tomoderate active proctitis and left-sided colitis can be treated with topical mesalamine alone or, in case of a mild-to-moderate pancolitis, in combination with oral mesalamine [9-11]. If mesalamine or other 5-ASA agents are ineffective, systemic steroid therapy should be used to try and induce remission [9]. Anti-TNFa antibodies or calcineurin inhibitors should be administered if no remission is achieved after 3-4 days. Vedolizumab has also been found effective against UC but should be reserved for less severe cases due to its late onset of effect [9]. To maintain remission, mesalamine should be given for at least 2 years [10]. Probiotics such as Escherichia coli Nissle have a similar efficacy as mesalamine for maintaining remission and can be given in case of mesalamine intolerance. Immunosuppressives should be administered in addition to aminosalicylates in case of frequent relapses, relapses despite the use of reserve medication, or steroid dependence [9]. After 3 months of immunosuppressive treatment, usually azathioprine or 6-mercaptopurine, steroid dose reduction should be attempted $[9,11]$. Anti-TNF $\alpha$ antibodies can be continued in order to maintain remission, and in general a combination of anti-TNFa antibodies with thiopurines is more effective than a monotherapy $[9,12,13]$. Vedolizumab, an integrin receptor antagonist, can be an alternative for refractory disease [14].

Modern Drug Therapy and Surgery for Ulcerative Colitis

\section{Influence of Drug Therapy on Surgery Rates and} Time of Surgery

Several studies have looked at the influence of biologicals on surgery rates by comparing the pre- to the post-biological era. Although most of the studies showed a decrease in surgery rates over time, no convincing surgery-sparing effect of newer medications has been proven [1]. A large population-based study from Denmark with 48,967 patients with IBD (Crohn's disease (CD): 13,185; UC: 35,782 ) assessed changes in surgery rates and medications during 1979-2011 [1]. Patients were separated into different time cohorts. The 5-year cumulative probability of first major surgery decreased from $44.7 \%$ in the earlier cohort (1979-1986) to $19.6 \%$ in the later cohort $(2003-2011)$ ( $\mathrm{p}<0.001)$ for CD, and from $11.7 \%$ in the earlier cohort (1979-1986) to 7.5\% in the later cohort (20032011) $(\mathrm{p}<0.001)$ for UC. From cohort $(1995-2002)$ to cohort (2003-2011), a significant increase in the use of thiopurines and TNF $\alpha$ blockers was observed, paralleled by a significant decrease in the use of 5-ASA and corticosteroids. Another study also analyzed the incidence of colectomies in UC patients before (19982004) and after (2005-2011) the introduction of biologics and found a significant decrease in colectomy rates [15]. Incidence rates for colectomy before and after introduction of biologicals were $36.08 / 1,000$ and $29.99 / 1,000$ patient years, respectively. Kaplan et al. [16] showed that over time, the use of purine anti-metabolites increased and elective colectomy rates decreased significantly in patients with UC. In contrast, emergent colectomy rates remained unchanged in this analysis [16]. However, due to the length of follow-up, it remains debatable whether the decrease in the rates of surgery will persist over time, or whether the need for surgery is just being postponed in patients exposed to immunosuppressive and biologics. Accordingly, another study demonstrated that about one-tenth of patients still require colectomy for UC at 5 years in the era of biologics [17]. Oral mesalamine (5-ASA), azathioprine, and anti-TNF therapy were not associated with a reduced need for colectomy. Thorne et al. [18] systematically reviewed the literature after 1990 reporting colectomy rates in UC patients treated with infliximab or cyclosporine. Infliximab was associated with a significantly lower colectomy rate than cyclosporine at 36 months but not at 3,12, or 24 months [18].

\section{Duration and Escalation of Conservative Treatment versus Surgery}

A therapy-refractory fulminant flare represents an emergency and needs to be treated in hospital. If there is no substantial improvement in disease intensity over a period of $72 \mathrm{~h}$ by conservative intensive care and drug treatment with high-dose steroids, surgery should be considered and interdisciplinarily discussed as an alternative to a further increase in drug therapy with calcineurin inhibitors or anti-TNFa antagonists $[11,19]$. Of note, a further increase in drug therapy using calcineurin inhibitors or anti-TNF $\alpha$ antibodies can postpone surgery for another year in approximately $42-65 \%$ 
of patients [20, 21]. Furthermore, independent analyses showed that delayed surgery in severe acute colitis results in significantly more major complications and higher mortality rates [22, 23]. Therefore, intensified drug therapy for fulminant colitis should only be conducted over a maximum period of 5-7 days, providing there is no deterioration of the patient. Deterioration during therapy requires urgent surgery within $24 \mathrm{~h}$ as it reduces mortality and morbidity $[11,24]$. If a toxic megacolon is present, the time window of a conservative therapy is significantly smaller and many of these patients may need emergency surgery [11]. The radiological crite-

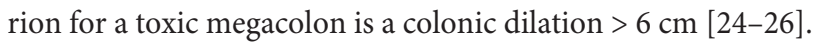

\section{Refractory Course}

A treatment-refractory course, despite the use of immunosuppressive drugs and/or biologics, represents an absolute indication for surgery [19]. Of these, the intractable fulminant flare should be distinguished as an emergency indication (see above). After failure of intensified, conservative therapy (immunosuppressants and/or biologics), surgical removal of the colon is indicated. In severe cases, delayed therapy leads to an increase in morbidity [27]. Therefore, adhering to an intensified conservative therapy should be limited in time. This is supported by a study which showed that from a retrospective point of view, more than $50 \%$ of patients would have preferred an earlier operation [28]. A recent metaanalysis on the usefulness of third-line therapies in severe chronic colitis showed that although short-term improvements are possible, the need for colectomy is only delayed, resulting in higher rates of complications [29].

\section{Elective Surgery or Continued Drug Therapy?}

Because of the effectiveness of modern medical treatment, the majority of patients with UC will not need to undergo surgery. However, there is a group of patients with therapy-refractory disease that will definitely require surgery in the long term. In those patients, biological therapy can only achieve short-term improvement and postpone the need for a colectomy. In addition to those who are refractory to medical treatment, patients suffering from persistent malaise, poor drug compliance, or carcinoma anxiety will particularly benefit from surgery [4-8]. Restorative proctocolectomy strives to remove the complete substrate of the disease and to re-establish gastrointestinal continuity. The majority of patients after IPAA can avoid hospitalizations and colitis-related medications, particularly immunosuppressants and immunomodulators and their associated potential adverse effects. In addition, the procedure substantially reduces UCrelated symptoms and the risk for dysplasia or cancer [19]. Also, surgical therapy has significantly evolved during the last decades. The introduction of laparoscopic surgery has led to a reduced length of hospital stay, reduction in symptoms of postoperative ileus, a lower rate of incisional hernias, and less wound infections. In addition, the minimally invasive procedure is associated with improved cosmetic results after IPAA. Therefore, surgery represents a good alternative to a long-term drug therapy. In the long term, despite the complications that can occur after IPAA, a good quality of life can be achieved for over $90 \%$ of all patients [4-8]. In a recently conducted case-control study there was no difference in quality of life between pouch patients versus patients receiving anti-TNFa therapy. However, pouch patients had higher quality of life scores, and the overall health care costs were lower [30]. In general, advanced age alone is not a contraindication for an IPAA. In view of the increased stool frequency due to relative sphincter weakness and a significantly higher postoperative morbidity, however, a permanent ileostomy should be considered in elderly patients, especially those over 65 years of age $[11,19]$.

\section{Influence of Modern Drug Therapy on Type of Surgery}

IPAA can be conducted as a one-, two- or three-step procedure. A one-stage operation should only be performed in selected patients with no signs of inflammation, good general condition, lowdose immunosuppressive therapy, and a totally tension-free IPAA [19]. In recent years, there has been a trend towards performing a three-step procedure [31]. The reason for this seems to be an intensified conservative therapy with increasing use of biologics. A three-stage procedure is conducted for emergency indications, in patients in an overall debilitated state of general health, or in patients with high and irreducible doses of immunosuppressive therapy [19]. The first-step procedure includes a subtotal colectomy and terminal ileostomy with Hartmann stump formation. After having recovered from that procedure, the patient can then have a completion proctectomy with IPAA. A temporary and protective loop ileostomy is usually performed. The loop ileostomy is then reversed in the third step. Overall, the three-stage procedure for patients with perioperative risk factors seems to be associated with a lower rate of complications than the one- or two-stage procedures [32-34]. However, Hicks et al. [35] showed in a retrospective analysis that in patients with active UC, use of steroids and anti-tumor necrosis factor agents alone does not appear to justify the decision to avoid IPAA creation at the first operation, provided that it is performed by a high-volume IBD surgeon. For patients with twostage procedures, they showed that the number of perioperative complications was affected by surgeon experience but not by emergent status, use of steroids, or anti-tumor necrosis factor agents. Two-stage procedures were associated with more perioperative complications but this difference was rather due to surgeon experience than to creation of an IPAA at the first operation [35].

A large current registry study from Denmark over the course of 33 years showed a significant association of a later pouch failure with the absence of a protective stoma [36]. In patients undergoing restorative proctocolectomy due to a refractory disease under immunosuppressive or immunomodulatory therapies, a stoma should generally be created as it effectively reduces the risk of pouch-related septic complications [37].
Kuehn/Hodin 


\section{Perioperative Medication and Complications}

Due to the heterogeneity of patients with IBD, with mostly multimodal or changing drug therapy, there is inconsistent, mostly retrospective data about perioperative medications [19]. Many studies do not differentiate between UC and CD but evaluate the perioperative risk in IBD in general.

There is a clear relationship between perioperative steroid medication and complication rates [38]. Taking $>20 \mathrm{mg}$ of prednisolone over a period of more than 2 weeks is associated with an increased complication rate of surgical therapy in $\operatorname{IBD}[11,39]$. Guidelines on UC suggest tapering of the glucocorticoid dose preoperatively, assuming this is clinically feasible [11]. Preoperative reduction to an equivalent of $<20 \mathrm{mg}$ prednisolone is desirable while avoiding the occurrence of a steroid withdrawal syndrome.

Meta-analyses about the risk of perioperative anti-TNFa therapy in IBD patients have shown increased infectious and non-infectious complication rates when limited to patients with CD [40]. This association could not be confirmed when numbers were limited to UC patients. When restricted to UC patients, both available meta-analyses found no significant influence of anti-TNFa on the perioperative complication rate when all types of surgical procedures were included $[41,42]$. However, the three-stage IPAA is more common in patients treated with anti-TNF $\alpha$ antibodies than in those without biologic therapy; thus, potential negative effects of these drugs on the perioperative complication rate could be underestimated $[11,43]$. In addition, in subgroup analyses for patients receiving IPAA, anti-TNFa therapy was significantly associated with early and late pouch-specific complications. This is consistent with two earlier studies that showed a significant association of anti-TNFa administration with perioperative complications up to 90 days before IPAA $[33,44]$. In addition, the odds of requirement for three-stage procedures was 2.07 times greater in the infliximab group [44]. A recent study from Kulaylat et al. [45], analyzing a health insurance database with 2,476 patients, confirmed this correlation; here, perioperative complications were significantly associated with the use of infliximab up to 90 days prior to pouch surgery. As no prospective randomized studies are available, it is not clear whether the increased complication rate in those studies may be due to the more severe underlying disease of patients treated with biologics [11]. Based on the available data, however, anti-TNFa therapy should be stopped prior to surgery. A safe time interval cannot be clearly recommended based upon available data. An interval of at least 4 weeks appears to be safe regarding the pharmacokinetic properties but might be difficult to implement in the clinical setting. Therefore, we think that a discontinuation of therapy should be aimed for at least 2 weeks before surgery. To what extent preoperative drug monitoring with measurement of anti-TNFa levels can contribute to determine a safe time point for surgery should be investigated in future studies.

Most studies did not show an influence of thiopurines on postoperative septic complications in UC but there is data about in- creased septic complications after thiopurine usage in CD [46]. As such, the benefit of stopping thiopurine therapy preoperatively remains debatable. Regarding immunosuppressive therapy with calcineurin inhibitors, a case series in children showed no differences in postoperative complications with or without preoperative therapy with these drugs [47]. A very recent meta-analysis on postoperative complications after preoperative use of vedolizumab in IBD patients did not appear to be associated with an increased risk of postoperative infectious or overall postoperative complications compared to either preoperative anti-TNF therapy or no biologic therapy [48]. Future studies on complications associated with UCspecific drugs should include perioperative drug level monitoring to improve the validity of the data. Finally, it is clear that interdisciplinary management regarding the best timing of surgery plays an indispensable role.

\section{New Therapeutic Approaches}

Many new substances including janus kinase inhibitors, antimucosal cell adhesion molecule 1 agents, anti-SMAD7 antisense oligonucleotides, anti-interleukin-12/23 monoclonal antibodies, and sphingosine-1-phosphate receptor-1 selective agonists are currently being evaluated for treatment of IBD [49]. Some of them will find their way into clinical practice but their overall benefit for patients is yet to be determined. Therefore, preclinical models with better reflection of human disease are needed. Recently, a humanized mouse model that mimics disease patterns of patients with UC has been successfully established [50]. Peripheral blood mononuclear cells of stratified patients with UC are injected in immunodeficient NOD/scid IL2R- $\gamma$ null mice. Colitis is induced by rectal ethanol application. This new model allows for testing of therapeutics directed against human targets and promises better translation of results to future clinical trials.

From the surgical point of view, an anti-inflammatory drug without having the negative side effects, including being a risk factor for surgical complications, would be preferable. The gut brush border enzyme intestinal alkaline phosphatase (IAP) functions as an anti-inflammatory epithelial defense factor that detoxifies a variety of proinflammatory byproducts such as lipopolysaccharides and CpG-DNA and promotes the gut barrier function [51-54]. IAP mRNA levels are reduced in patients with UC [55]. IAP also promotes growth of intestinal commensal bacteria, preserving homeostasis of the gut microbiota $[53,56]$. As a naturally occurring enzyme, IAP has a low risk profile $[57,58]$. The safety and efficacy were evaluated in an open-label trial with application of IAP via a nasoduodenal tube. After a 7-day treatment, the drug showed clinical response in $48 \%$ of patients with moderate to severe disease and was well tolerated [58]. However, there is a need for an acid-resistant oral form of this enzyme in order to further evaluate its role as an effective and well-tolerated therapeutic for UC. 


\section{Conclusion}

In recent years, modern drug therapy has changed the timing, approach, and outcomes of surgery for UC. Most of the studies showed a decrease in surgery rates over time while the rate of emergency colectomies remained unchanged. So far, no convincing surgery-sparing effect of newer medications was found, and it remains debatable if surgery rates decreased because of improved management for UC or due to the introduction of biologicals. Due to an intensified conservative therapy, there has been a trend towards performing a three-step procedure in the last decade. A subset of patients with complex refractory course of disease are most likely to benefit from elective IPAA as an alternative to prolonged conservative therapies after failure of first-line treatment. Surgery should not be considered as the negative endpoint of treatment modalities but as a good alternative to a prolonged conservative therapy for some patients. In conclusion, a close cooperation between the various disciplines in the pre- and postoperative management is essential for an optimal timing and outcome of patients with UC.

\section{Disclosure Statement}

The authors have declared that no conflict of interest exists.

\section{References}

1 Rungoe C, Langholz E, Andersson M, et al: Changes in medical treatment and surgery rates in inflammatory bowel disease: a nationwide cohort study 1979-2011. Gut 2014;63:1607-1616.

${ }_{2}$ Frolkis $\mathrm{AD}$, et al: Risk of surgery for inflammatory bowel diseases has decreased over time: a systematic review and meta-analysis of population-based studies. Gastroenterology 2013;145:996-1006.

3 Cohen JL, Strong SA, Hyman NH, et al: Practice parameters for the surgical treatment of ulcerative colitis. Dis Colon Rectum 2005;48:1997-2009.

4 Fazio VW Kiran RP, Remzi FH, et al: Ileal pouch anal anastomosis: analysis of outcome and quality of life in 3707 patients. Ann Surg 2013;257:679-685.

5 Lichtenstein GR, Cohen R, Yamashita B, et al: Quality of life after proctocolectomy with ileoanal anastomosis for patients with ulcerative colitis. J Clin Gastroenterol 2006;40:669-677.

6 Heuschen UA, Heuschen G, Rudek B, et al: Long-term quality of life after continence-preserving proctocolectomy for ulcerative colitis and familial adenomatous polyposis (Article in German). Chirurg 1998;69:10521058.

7 Heikens JT, de Vries J, van Laarhoven CJ: Quality of life, health-related quality of life and health status in patients having restorative proctocolectomy with ileal pouch-anal anastomosis for ulcerative colitis: a systematic review. Colorectal Dis 2012;14:536-544.

8 Ikeuchi H, et al: Surgery for ulcerative colitis in 1,000 patients. Int J Colorectal Dis 2010;25:959-965.

$\checkmark 9$ Wehkamp J, Götz M, Herrlinger K, et al: Inflammato bowel disease. Dtsch Arztebl Int 2016;113:72-82.

10 Feagan BG, Macdonald JK: Oral 5-aminosalicylic acid for maintenance of remission in ulcerative colitis. Cochrane Database Syst Rev 2012;10:CD000544.

11 Dignass A, Preiss JC, Aust DE, et al: Updated German guideline on diagnosis and treatment of ulcerative colitis (Article in German). Z Gastroenterol 2011;49:1276341.

12 Danese S, Fiorino G, Peyrin-Biroulet L, et al: Biological agents for moderately to severely active ulcerative colitis: a systematic review and network meta-analysis. Ann Intern Med 2014;20:160:704-711.

13 Panaccione R, Ghosh S, Middleton S, et al: Combination therapy with infliximab and azathioprine is superior to monotherapy with either agent in ulcerative colitis. Gastroenterology 2014;146:392-400.

14 Feagan BG, Rutgeerts P, Sands BE, et al: Vedolizumab as induction and maintenance therapy for ulcerative colitis. N Engl J Med 2013;369:699-710.
5 Abou Khalil M, Boutros M, Nedjar H, et al: Incidence rates and predictors of colectomy for ulcerative colitis in the era of biologics: results from a provincial database. J Gastrointest Surg 2018;22:124-132.

16 Kaplan GG, Seow CH, Ghosh S, et al: Decreasing colectomy rates for ulcerative colitis: a populationbased time trend study. Am J Gastroenterol 2012;107: 1879-1887.

17 Williet N, Pillot C, Oussalah A, et al: Incidence of and impact of medications on colectomy in newly diagnosed ulcerative colitis in the era of biologics. Inflamm Bowel Dis 2012;18:1641-1646.

18 Thorne K, Alrubaiy L, Akbari A, et al: Colectomy rates in patients with ulcerative colitis following treatment with infliximab or ciclosporin: a systematic literature review. Eur J Gastroenterol Hepatol 2016;28:369-382.

19 Kühn F, Klar E: Surgical principles in the treatment of ulcerative colitis. Viszeralmedizin 2015;31:246-250.

20 Actis GC, Fadda M, David E, et al: Colectomy rate in steroid-refractory colitis initially responsive to cyclosporin: a long-term retrospective cohort study. BMC Gastroenterol 2007;7:13.

21 Croft A, Walsh A, Doecke J, et al: Outcomes of salvage therapy for steroid-refractory acute severe ulcerative colitis: ciclosporin vs. infliximab. Aliment Pharmacol Ther 2013;38:294-302.

22 Randall J, Singh B, Warren BF, et al: Delayed surgery for acute severe colitis is associated with increased risk of postoperative complications. Br J Surg 2010;97: 404-409.

23 Kaplan GG, McCarthy EP, Ayanian JZ, et al: Impact of hospital volume on postoperative morbidity and mortality following a colectomy for ulcerative colitis. Gastroenterology 2008;134:680-687.

24 Heppell J, Farkouh E, Dube S, et al: Toxic megacolon. An analysis of 70 cases. Dis Colon Rectum 1986;29: 789-792.

25 Gan SI, Beck PL: A new look at toxic megacolon: an update and review of incidence, etiology, pathogenesis, and management. Am J Gastroenterol 2003;98:2363-2371.

26 Sheth SG, LaMont JT: Toxic megacolon. Lancet 1998; 351:509-513.

27 Kimura H, Kunisaki R, Tatsumi K, et al: Prolonged medical therapy increases the risk of surgical complications in patients with severe ulcerative colitis. Dig Surg 2016;33:182-189.

28 Neumann PA, Mennigen RB, Senninger N, et al: Timing of restorative proctocolectomy in patients with medically refractory ulcerative colitis: the patient's point of view. Dis Colon Rectum 2012;55:756-761.
29 Feuerstein JD, Akbari M, Tapper EB, et al: Systematic review and meta-analysis of third-line salvage therapy with infliximab or cyclosporine in severe ulcerative colitis. Ann Gastroenterol 2016;29:341-347.

30 van der Valk ME, Mangen MJ, Severs M, et al: Comparison of costs and quality of life in ulcerative colitis patients with an ileal pouch-anal anastomosis, ileostomy and anti-TNFalpha therapy. J Crohns Colitis 2015;9:1016-1023.

31 Devaraj B, et al: Surgical management of ulcerative colitis in the era of biologicals. Inflamm Bowel Dis 2015; 21:208-220.

32 Nicholls RJ, Holt SD, Lubowski DZ: Restorative proctocolectomy with ileal reservoir. Comparison of twostage vs. three-stage procedures and analysis of factors that might affect outcome. Dis Colon Rectum 1989;32: 323-326.

33 Selvasekar CR, Cima RR, Larson DW, et al: Effect of infliximab on short-term complications in patients undergoing operation for chronic ulcerative colitis. J Am Coll Surg 2007;204:956-962.

34 Stewart D, Chao A, Kodner I, et al: Subtotal colectomy for toxic and fulminant colitis in the era of immunosuppressive therapy. Colorectal Dis 2009;11:184-190.

35 Hicks CW, Hodin RA, Savitt L, et al: Does intramesorectal proctectomy with rectal eversion affect postoperative complications compared to standard total mesorectal excision in patients with ulcerative colitis? J Gastrointest Surg 2014;18:385-90.

36 Mark-Christensen A, Erichsen R, Brandsborg S, et al: Pouch failures following ileal pouch-anal anastomosis for ulcerative colitis. Colorectal Dis 2018;20:44-52.

37 Mennigen R, Sewald W, Senninger N, et al: Morbidity of loop ileostomy closure after restorative proctocolectomy for ulcerative colitis and familial adenomatous polyposis: a systematic review. J Gastrointest Surg 2014;18:2192-2200.

38 Subramanian V, et al: Preoperative steroid use and risk of postoperative complications in patients with inflammatory bowel disease undergoing abdominal surgery. Am J Gastroenterol 2008;103:2373-2381.

39 Kühn F, Nixdorf M, Schwandner F, et al: Risk factors for early surgery and surgical complications in Crohn's disease (Article in German). Zentralbl Chir 2018;143: 11-17.

40 Narula N, et al: Meta-analysis: peri-operative anti$\mathrm{TNF}$ a treatment and post-operative complications in patients with inflammatory bowel disease. Aliment Pharmacol Ther 2013;37:1057-1064. 
41 Yang Z, Wu Q, Wang F, et al: Meta-analysis: effect of preoperative infliximab use on early postoperative complications in patients with ulcerative colitis undergoing abdominal surgery. Aliment Pharmacol Ther 2012;36:922-928.

42 Selvaggi F, Pellino G, Canonico S, et al: Effect of preoperative biologic drugs on complications and function after restorative proctocolectomy with primary ileal pouch formation: systematic review and meta-analysis. Inflamm Bowel Dis 2015;21:79-92.

43 Norgard BM, Nielsen J, Qvist N, et al: Pre-operative use of anti-TNF-alpha agents and the risk of post-operative complications in patients with ulcerative colitis - a nationwide cohort study. Aliment Pharmacol Ther 2012;35:1301-1309.

44 Mor IJ, Vogel JD, da Luz Moreira A, et al: Infliximab in ulcerative colitis is associated with an increased risk of postoperative complications after restorative proctocolectomy. Dis Colon Rectum 2008;51:1202-1207; discussion 1207-1210.

45 Kulaylat AS, Kulaylat AN, Schaefer EW, et al: Association of preoperative anti-tumor necrosis factor therapy with adverse postoperative outcomes in patients undergoing abdominal surgery for ulcerative colitis. JAMA Surg 2017;152:e171538.
Myrelid P, et al: Thiopurine therapy is associated with postoperative intra-abdominal septic complications in abdominal surgery for Crohn's disease. Dis Colon Rectum 2009;52:1387-1294.

47 Hait EJ, Bousvaros A, Schuman M, et al: Pouch outcomes among children with ulcerative colitis treated with calcineurin inhibitors before ileal pouch anal anastomosis surgery. J Pediatr Surg 2007;42:31-34; discussion 34-35.

48 Law CCY, Narula A, Lightner AL, et al: Systematic review and meta-analysis: preoperative vedolizumab treatment and postoperative complications in patients with inflammatory bowel disease. J Crohns Colitis. 2018;12:538-545.

49 Lee HS, Park SK, Park DI: Novel treatments for inflammatory bowel disease. Korean J Intern Med 2018; 33:20-27.

50 Palamides $\mathrm{P}$, Jodeleit H, Föhlinger M, et al: A mouse model for ulcerative colitis based on NOD-scid IL2R $\gamma$ null mice reconstituted with peripheral blood mononuclear cells from affected individuals. Dis Model Mech 2016;9:985-997.

51 Chen KT, Malo MS, Moss AK, et al: Identification of specific targets for the gut mucosal defense factor intestinal alkaline phosphatase. Am J Physiol Gastrointest Liver Physiol 2010;299:G467-475.

52 Liu W, Hu D, Huo H, et al: Intestinal alkaline phosphatase regulates tight junction protein levels. J Am Coll Surg 2016;222:1009-1017.
53 Hamarneh SR, Mohamed MM, Economopoulos KP, et al: A novel approach to maintain gut mucosal integrity using an oral enzyme supplement. Ann Surg 2014;260: 706-714; discussion 714-715.

54 Ramasamy S, Nguyen DD, Eston MA, et al: Intestinal alkaline phosphatase has beneficial effects in mouse models of chronic colitis. Inflamm Bowel Dis 2011;17: 532-542.

55 Tuin A, Poelstra K, de Jager-Krikken A, et al: Role of alkaline phosphatase in colitis in man and rats. Gut 2009;58:379-387.

56 Malo MS, Moaven O, Muhammad N, et al: Intestinal alkaline phosphatase promotes gut bacterial growth by reducing the concentration of luminal nucleotide triphosphates. Am J Physiol Gastrointest Liver Physiol 2014;306:826-838.

57 Pickkers P, Heemskerk S, Schouten J, et al: Alkaline phosphatase for treatment of sepsis-induced acute kidney injury: a prospective randomized double-blind placebo-controlled trial. Crit Care 2012;16:R14.

58 Lukas M, Drastich P, Konecny M, Gionchetti P, Urban O, Cantoni F, Bortlik M, Duricova D, Bulitta M: Exogenous alkaline phosphatase for the treatment of patients with moderate to severe ulcerative colitis. Inflamm Bowel Dis 2010;16:1180-116. 